\title{
Genes, the environment and the control of food intake
}

\author{
John M. de Castro* \\ Department of Psychology, University of Texas at El Paso, El Paso, TX 79968, USA
}

\begin{abstract}
Genes are known to have independent influences on the height and weight of individuals, their overall levels of nutrient intakes and their meal sizes and frequencies. Recent evidence suggests that genes exert multiple and subtle influences on the controls of food intake. There are significant genetic influences on the level and responsiveness of the individual to physiological factors, such as the preprandial stomach contents of nutrients and subjective hunger, and also to environmental and psychological factors such as social facilitation of eating, diurnal rhythms of intake, palatability, cognitive restraint and dietary density. The general model of intake regulation provides an integrated and comprehensive account of how these physiological and environmental factors might fit together to produce the control of intake and body weight.
\end{abstract}

Genes: Environment: Obesity

It is a precept of the behavioural sciences that behaviour is the outcome of the joint action of nature and nurture: the genes and the environment. The genes act to determine the anatomy and physiology of the individual, while the environment acts upon that physiology to produce behaviour. This rather simple but elegant view has guided research on ingestive behaviour. There has been little research, however, on the interaction between the genes and the environment. There are considerable interactions, such that the impact of the environment upon intake depends upon inherited susceptibilities. In addition, inheritance influences the nature of the environments that the individual seeks out and prefers. Hence, the genes, in part, determine the environment. Genes, the environment and their interaction act in concert to control ingestive behaviour.

\section{Genes}

Considerable evidence has been amassed on the genetic influence on body size, body composition and even on the metabolic response to feeding, including the tendency to store energy as lean tissue or fat. If body size is influenced by inheritance, then the ingestive behaviours that underlie the growth and maintenance of body mass should also be influenced by genes. Indeed, twin studies have demonstrated that heredity accounts for $42 \%$ of the variance in average overall daily intakes (de Castro, 1993a). Surprisingly, this influence is independent of body size as the effect is still highly significant after accounting for the effects of height, weight, gender and age (de Castro, $1993 b$ ). If overall daily intake is influenced by inheritance, then the meal intakes that underlie total intake should also be influenced by the genes. Indeed, heredity accounts for $28 \%$ of the variance in the meal sizes and $34 \%$ of the variance in the meal frequencies of twins, even after accounting for the influence of overall daily intake. Hence, the genes have separate and independent effects on height, weight, overall intake, meal size and meal frequency.

Physiology can affect meal intakes by influencing gastrointestinal physiology, stomach capacity and emptying, and thus can affect the amount eaten. The nutrients remaining in the stomach at any point in time can be estimated with a simple equation that uses the amount eaten in meals and the passage of time as an indicator of stomach emptying. Significant genetic influences are observed on the amount that an individual tends to have in the stomach before and after a meal (Fig. 1(A)). Some individuals tend to eat their meals with the stomach relatively empty, while others with it relatively full, and this individual difference is affected by inheritance (de Castro, 1999b). The stomach content is a significant influence on intake as evidenced by the significant negative correlations between the beforemeal stomach content and meal size (de Castro et al. 1986). Genetic factors also affect the degree of restraint on the amount eaten exerted by stomach filling. The responsiveness of the individual to the stomach content can be estimated with the slope of the relationship between the amount in the stomach and the amount eaten, and this is significantly heritable (Fig. 1(B)). Hence, heredity influences how full the individual is when they begin to eat and how great a suppressive effect stomach content will have on their subsequent intake.

Stomach filling also affects the subjective, psychological state of hunger, and hunger is strongly correlated with the meal size, with the greater the level of hunger the more that will be eaten and the more that is eaten the lower the level of hunger (de Castro, 1999a). The level of hunger at which an individual initiates a meal is heritable. Analysis of the twin data revealed significant genetic influences on the 


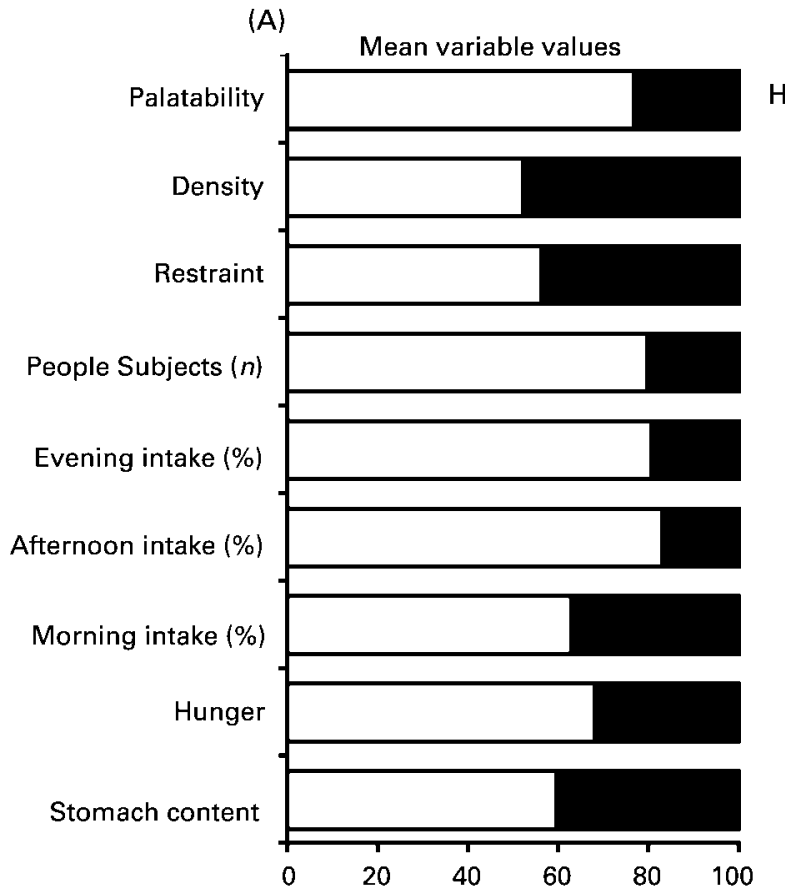

Proportion of the variance accounted for $(\%)$
(B)

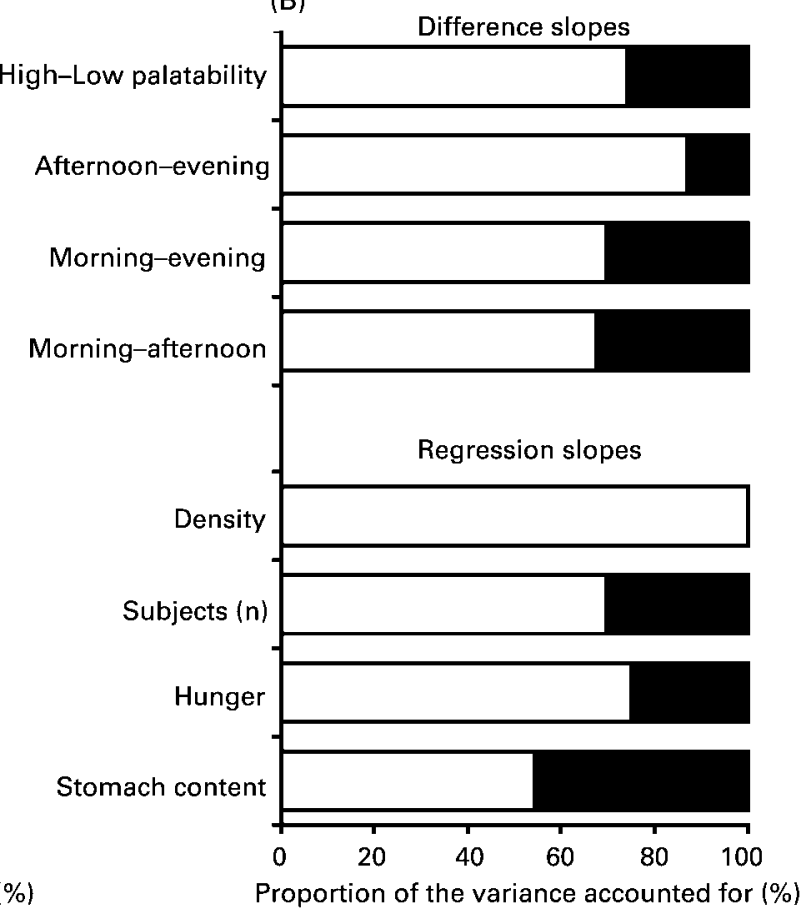

Fig. 1. The proportion of the variance in the factor means $(A)$ and the slopes of the relationships between the factors and the meal size (B) that could be accounted for by the individual environment $(\square)$, family environment $($ 目) and heredity $(\square)$ in the linear structural modelling heritability analysis of the data from twins. (From de Castro 1997, 1999a,b, 2001a,b, J de Castro, unpublished results.)

levels of hunger both before and after the meal (Fig. 1(B); de Castro, 1999a). In addition, genetic factors affect the increase in the amount eaten produced by hunger. Significant heritabilities are present for both the correlation and the slope of the regression between hunger and meal size (Fig. 1(B)). Hence, there are inherited individual differences in the impact that hunger has on the meal size. In addition, the relationship between the amount ingested in the meal and the change in hunger produced is influenced by inheritance. Significant heritabilities were obtained for both the correlation and the slope of the regression between meal size and the hunger change, indicating that there are inherited individual differences in the effect of nutrient ingestion on subsequent hunger. Thus, heredity influences how hungry the individual is when they begin to eat and how big of a facilitative effect hunger has on subsequent intake, how hungry the individual is when they have finished eating, and how big of an impact intake has on changing perceived hunger.

\section{The environment}

There is a considerable amount of variance in intake that is not accounted for by heredity. Inheritance accounts for $42 \%$ of the variance in overall intake, while $58 \%$ is due to environment; inheritance accounts for 28 and $34 \%$ of the variance in meal size and frequency, while 72 and $64 \%$ are due to environment respectively.

Man is a social animal whose behaviour, including food intake, is profoundly affected by social influences. Meals eaten with other people present are $44 \%$ larger than meals eaten alone (de Castro \& de Castro, 1989). The presence of other people acts by extending the amount of time spent at a meal and thus increasing the amount eaten (de Castro, 1990). This is an orderly phenomenon, with the more people present the more that is ingested. One other person present at the meal was associated with a $33 \%$ increase in meal size while 47, 58, 69, 70, 72 and $96 \%$ increases were associated with two, three, four, five, six and seven or more people respectively (de Castro \& Brewer, 1992).

There are clear diurnal influences on intake. As the day progresses meal sizes increase (de Castro, 1987), while the following interval until the next meal gets shorter. The satiety ratio (inter-meal interval:meal size) gauges the period of satiety produced per unit food-energy ingested. It shows a marked decline over the day, becoming quite low by late evening. Thus, the satiating effect of food decreases over the course of the day. Indeed, eating a large proportion of intake in the morning is associated with lower overall intake, while eating a high proportion of intake in the evening is associated with higher overall intake (de Castro, 2004).

Psychological phenomena are major influences on intake. Human subjects differ in the degree to which they attempt to assert control over their food intake. Dietary restraint has been shown to be associated with lower overall intakes and the ingestion of smaller meals, lower in fat (de Castro, 1995). Palatability is a hypothetical construct that stands for the stimulus qualities of a substance that affects its acceptability (Rogers, 1990). Palatability has been shown to have a positive relationship with the amount ingested, with meals $44 \%$ larger when the food was rated highly palatable than when the food was neutral or unpalatable (de Castro et al. 2000).

If the weight of the food and not its energy content is regulated, then the density of the diet ingested would be a major factor determining total energy intake, and in 
turn body weight and adiposity. Indeed, diet density has been repeatedly shown to be significantly associated with intake (Yao \& Roberts, 2001). The density of meals or the total daily intake are strongly correlated with their total energy content; the greater the density the more energy ingested ( $\mathrm{J}$ de Castro, 2004b). Disappointingly, however, diet density differences were not related to body weight or BMI differences, and dietary density is not correlated with body weight or BMI. Hence, dietary density affects short-term meal-to-meal and day-to-day intake, but it does not appear to have long-term effects on body weight and fatness.

\section{Gene-environment interactions}

It is clear that intake is affected by the genes through the determination of physiological structure and that the environment also has a major influence on intake. To ascertain whether inheritance affects environmental influences on intake, genetic influences on the relationship of environmental factors with intake were investigated.

\section{Inheritance influences social effects on intake}

Analysis of data from twins has revealed heritable individual differences in the number of eating companions at meals, accounting for more than $20 \%$ of the variance (Fig. 1(B); de Castro, 1997). Heredity also influences the nature of the companions, accounting for more than $25 \%$ of the variance in the likelihood of eating with family, friends and spouse. In addition, heredity affects how much intake is increased by the presence of other people. Both the correlation and the slope of the regression were significantly heritable (Fig. 1(B)). This indicates that genetic factors affect the number and types of people at meals and the impact of these companions on intake. This is quite remarkable, given that the number of other people present would, on the surface, appear to be primarily due to environmental conditions.

Again employing the twin data, the influence of heredity on the time of day and its relationship with intake was investigated. Some people eat a larger portion of their daily intake than others in the morning, some in the afternoon and some in the evening, and these proportions are heritable (Fig. 1(A); de Castro 2001a). This indicates that individual differences in the time of day when people eat are influenced by heredity. In addition, the differences in the proportions of intake between the morning and afternoon, the morning and evening, and the afternoon and evening were significantly heritable (Fig. 1(A)). This indicates that genetic factors affect when an individual eats and the impact of that time selection on intake.

To look at the influence of the genes on psychological factors, the measured levels of restraint in the twins was subjected to heritability analysis. It was discovered that there were significant genetic effects that accounted for $44 \%$ of the variance in restraint (Fig. 1(B); J de Castro and L Lilenfeld, unpublished results). The inheritance of palatability-food intake relationships was also studied in the twins. Inheritance accounted for $23 \%$ of the variance in the before-meal palatability ratings, indicating that the genes influence the meal's perceived palatability (Fig. 1(B); de Castro, 2001b). Similarly, genes influenced the amounts ingested in either low palatability or high palatability meals. The difference in the amount ingested between low and high palatable meals, a metric of the responsiveness of the individual to palatability, was also significantly heritable (Fig. 1(A)). Hence, genes influence how much is eaten in a meal, the preferred palatability and the reactivity of the individual to that palatability.

The data from twins were analysed to ascertain the influence of the genes on dietary density effects. Dietary density is a highly heritable factor with inheritance accounting for more than $40 \%$ of the variance (Fig. 1(B); $\mathrm{J}$ de Castro, unpublished results). On the other hand, heredity did not influence the relationship between density and intake. Neither the correlation nor the slope of the regression between density and intake was heritable (Fig. 1(A)). Hence, heredity influences the preferred diet density that in turn has a major influence on intake. But individual differences in responsiveness to density are not due to heredity.

\section{A general model of intake regulation}

Intake is affected by a wide range of physiological and environmental factors, each of which only accounts for a small portion of the variance in intake. In addition, the level and impact of these various factors can vary from individual to individual and these individual differences are affected by heredity. These elements were incorporated into a general model of intake regulation (de Castro \& Plunkett, 2002) presented in Fig. 2. The model incorporates sets of uncompensated (primarily environmental) and compensated (primarily physiological) factors that have preferred levels that are influenced by heredity. Further, the model

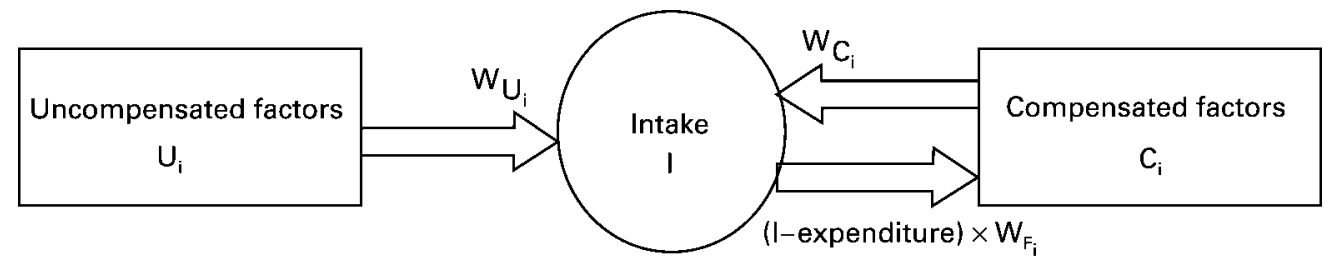

Fig. 2. The general intake regulation model. Intake $(\mathrm{I})$ is controlled by two sets of factors: compensated factors $\left(\mathrm{C}_{\mathrm{i}}\right)$ that both affect and are affected by intake via negative feedback loops; uncompensated factors $\left(U_{i}\right)$ that affect but are not affected by intake. Inheritance affects the system by determining the preferred level for intake, and compensated and uncompensated factors and also by determining the level of impact of the factors on intake (W). Factors that are affected by heredity are. (From de Castro \& Plunkett, 2002.) 
specifies an individual impact factor, weight, for each factor that specifies the magnitude of the factor's effect on intake. These weights are values between -1 and +1 and are assumed to differ between individuals and be affected by heredity.

A computer simulation was implemented to test the model's response to changes such as those that occur in the natural environment and individual differences in responsiveness to environmental changes. To test the model's response to an increase in the level of an uncompensated environmental factor the level of one of the uncompensated factors was doubled. In response to the change, the model indicated a rapid rise in body weight. At first the body weight became unstable and oscillated, but then settled at a $7 \%$ greater body weight. This new body weight was maintained as long as no further changes occurred. To investigate how the model responds to individual responsiveness differences, the weighting factor was manipulated along with the uncompensated factor. Doubling of the uncompensated factor with a low weight produced a much smaller increase in body weight than when a larger weight was used. Body weight depended upon both the level of the uncompensated factor and its weighting.

\section{Discussion}

If food intake can be considered a model behaviour that is representative of behaviour in general, then it is clear that the control of behaviour is a complex integral of a large number of influences. This complexity is well represented in the general model of intake regulation. Some of these factors originate from the environment, some from heredity, but many from the interaction of heredity and environment. Clearly, the environment has major effects upon intake. In addition, genes influence the anatomy and physiology of the individual that is essential for the control of intake. However, the genes can also influence the environment and its impact on intake.

It may seem strange to postulate that heredity influences the environment. But there are inborn proclivities to seek out or maintain particular environments at particular levels. This is demonstrated by the significant inheritances of the number of people present, the time of day of eating and the density and palatability of the diet. Since the genes directly affect only the individual's physiology, there must be physiological intermediaries underlying these environmental inheritances. Inborn differences in personality characteristics, such as extraversion (Saudino et al. 1997), in circadian oscillators (Kolker \& Turek, 1999) or in the gustatory system (Matsunami et al. 2000) could explain how the genes affect the environment.

It is concluded that food intake in free-living human subjects is controlled by a wide range of genetic, physiological, psychological, social and cultural variables. Each of these factors is characterized by large individual differences in both level and responsiveness. The proposed general model of intake regulation provides an integrated and comprehensive accounting of how all these pieces might fit together to produce the control of intake.

\section{Acknowledgements}

Supported in part by a Georgia State University Research Program Enhancement Grant.

\section{References}

de Castro JM (1987) Circadian rhythms of the spontaneous meal patterns, macronutrient intake, and mood of humans. Physiol Behav 40, 437-466.

de Castro JM (1990) Social facilitation of duration and size but not rate of the spontaneous meal intake of humans. Physiol Behav 47, 1129-1135.

de Castro JM (1993a) Genetic influences on daily intake and meal patterns of humans. Physiol Behav 53, 777-782.

de Castro JM (1993b) Independence of genetic influences on body size, daily intake, and meal patterns of humans. Physiol Behav 54, 633-639.

de Castro JM (1995) The relationship of cognitive restraint to the spontaneous food and fluid intake of free-living humans. Physiol Behav 57, 287-295.

de Castro JM (1997) Inheritance of social influences on eating and drinking in humans. Nutr Res 17, 631-648.

de Castro JM (1999a) Inheritance of hunger relationships with food intake in free living humans. Physiol Behav 67, 249-258.

de Castro JM (1999b) Inheritance of pre-meal stomach content influences on eating and drinking in free-living humans. Physiol Behav 66, 223-232.

de Castro JM (2001a) Heritability of diurnal changes in food intake in free-living humans. Nutrition 17, 713-720.

de Castro JM (2001b) Palatability and intake relationships in free-living humans: influence of heredity. Nutr Res 21, 935-945.

de Castro JM (2004a) The time of day of food intake influences overall intake in humans. J Nutr 134, 104-111.

de Castro JM (2004b) Density and intake relationships in the eating behavior of free-living humans. J Nutr 134, 335-341.

de Castro JM, Bellisle F, Dalix AM \& Pearcey S (2000) Palatability and intake relationships in free-living humans: characterization and independence of influence in North Americans. Physiol Behav 70, 343-350.

de Castro JM \& Brewer EM (1992) The amount eaten in meals by humans is a power function of the number of people present. Physiol Behav 51, 121-125.

de Castro JM \& de Castro ES (1989) Spontaneous meal patterns in humans: influence of the presence of other people. Am J Clin Nutr 50, 237-247.

de Castro JM, McCormick J, Pedersen M \& Kreitzman SN (1986) Spontaneous human meal patterns are related to preprandial factors regardless of natural environmental constraints. Physiol Behav 38, 25-29.

de Castro JM \& Plunkett S (2002) A general model of intake regulation. Neurosci Biobehav Rev 26, 581-595.

Kolker DE \& Turek FW (1999) The search for circadian clock and sleep genes. J Psychopharmacol 13, Suppl. 1, S5.

Matsunami H, Montmayeur JP \& Buck LB (2000) A family of candidate taste receptors in human and mouse. Nature 404, 601-602.

Rogers PJ (1990) Why a palatability construct is needed. Appetite 14, 167-170.

Saudino KJ, Pedersen NL, Lichtenstein P, McClearn GE \& Plomin R (1997) Can personality explain genetic influences on life events? J Pers Soc Psychol 72, 196-206.

Yao M \& Roberts SB (2001) Dietary energy density and weight regulation. Nutr Rev 59, 247-258. 